\title{
ИЗМЕРЕНИЕ ПЕРИМЕТРА И ПЛОЩАДИ ЛИСТА ДЕРЕВА
}

\author{
А.И. Кудряшова \\ Поволжский государственный технологический университет, \\ Россия, г. Йошкар-Ола
}

Введение. Техническое решение относится к инженерной биологии и биоиндикации окружающей среды измерениями качества ростовых органов различных видов растений, преимущественно древесных растений, например, проб в виде листьев древесных растений с простой и небольшой листовой пластинкой: липы, клена полевого или американского, березы, тополя.

Предлагаемое техническое решение может быть также использовано при экологическом и технологическом мониторинге за молодыми древесными растениями, в частности, за молодняками лесных деревьев, а также за качеством городских парков и других насаждений, естественного облесения пустырей, бывших строительных и иных площадок рекультивации земель, залежей земель сельскохозяйственного назначения.

Методика эксперимента. Опыты были проведены в городе Йошкар-Оле 21.05.2014 и 29.05.2014 на одних и тех же 10 листах липы, расположенной на углу перекрестка по улицам Машиностроителей и Красноармейской.

Способ измерения площади листьев липы по аналогу [1] и прототипу [2] включает такие действия: лист растения располагают на белой бумаге, затем кладут сверху прозрачную палетку с сеткой, например, с малыми клетками размерами $2 \times 2$ мм, так, чтобы средняя линия вдоль палетки совпала с осью продольной жилки листа. Затем лист с палеткой фотографируют (фиг.1), например, цифровым фотоаппаратом с функциями фотографирования и хранения множества фотоснимков в памяти

У каждого листа взять его фото и подрезать на компьютере лишние кромки, а затем увеличить для удобства подсчетов клеток до формата A4.

Исходные данные по замерам приведены в таблице 1 . Измерить длину $a$ и ширину $b$ листа очень просто палеткой по числу сантиметровых клеток и дополнением малых клеток, а в концах изображения и по долям малых клеток.

В таблицах 1 (на 21.05.2014) и 2 (на 29.05.2014) даны следующие условные обозначения физическим параметрам листа:

$a$ - длина листа вдоль главной жилки, измеряемая от места соединения черешка с листовой пластиной растения до конца вершинки листа, мм;

$b$ - ширина листа по крайним точкам поперек листовой пластины, или же общая ширина листа в наибольшем поперечном сечении листа, мм;

$P$ - периметр листа, см;

$S$ - площадь листа, измеряемая количеством клеток размерами $2 \times 2$ мм и количеством клеток периметра листа, см² $^{2}$.

После этого периметр и площадь вычисляют по формулам:

$$
\begin{gathered}
P=0,1 \sqrt{2^{2}+2^{2}} I_{P}=0,1 \sqrt{8} I_{P}=0,28284 I_{P} ; \\
S=0,04 I_{S}+0,02 I_{P} .
\end{gathered}
$$


Таблица 1

Количество клеток, периметр, площадь и расчетные параметры листа

\begin{tabular}{|c|c|c|c|c|c|c|c|c|c|c|}
\hline \multirow[b]{2}{*}{$\begin{array}{c}\text { № } \\
\text { ח/П }\end{array}$} & \multicolumn{10}{|c|}{21.05 .2014} \\
\hline & $\begin{array}{l}I_{P}, \\
\text { шт. }\end{array}$ & $\begin{array}{l}I_{S}, \\
\text { шт. }\end{array}$ & $\begin{array}{l}P \\
\mathrm{~cm}\end{array}$ & $\begin{array}{c}S, \\
\mathrm{Cm}^{2}\end{array}$ & $\begin{array}{l}K \\
\mathrm{~cm}\end{array}$ & $k$ & $\begin{array}{c}a, \\
\text { MМ }\end{array}$ & $\begin{array}{l}b, \\
\text { MM }\end{array}$ & $k_{0}$ & $\vartheta$ \\
\hline 1 & 53 & 241 & 14,99 & 10,70 & 0,7138 & 0,0476 & 36 & 40 & 0,7431 & 0,9000 \\
\hline 2 & 43 & 278 & 12,16 & 11,98 & 0,9850 & 0,0810 & 42 & 40 & 0,7131 & 1,0500 \\
\hline 3 & 61 & 370 & 17,25 & 16,02 & 0,9285 & 0,0538 & 50 & 46 & 0,6965 & 1,0870 \\
\hline 4 & 46 & 498 & 13,01 & 20,84 & 1,6018 & 0,1231 & 54 & 44 & 0,8771 & 1,2273 \\
\hline 5 & 60 & 495 & 16,97 & 21,00 & 1,2374 & 0,0729 & 50 & 60 & 0,7000 & 0,8333 \\
\hline 6 & 63 & 509 & 17,82 & 21,62 & 1,2133 & 0,0681 & 46 & 58 & 0,8103 & 0,7931 \\
\hline 7 & 67 & 495 & 18,95 & 21,14 & 1,1156 & 0,0589 & 54 & 46 & 0,8510 & 1,1739 \\
\hline 8 & 45 & 551 & 12,73 & 22,94 & 1,8024 & 0,1416 & 54 & 56 & 0,7586 & 0,9643 \\
\hline 9 & 51 & 604 & 14,42 & 25,18 & 1,7456 & 0,1210 & 60 & 48 & 0,8743 & 1,2500 \\
\hline 10 & 47 & 500 & 13,29 & 20,94 & 1,5752 & 0,1185 & 58 & 50 & 0,7221 & 1,1600 \\
\hline
\end{tabular}

По предлагаемому способу подсчитывают количество клеток:

$I_{P}$ - количество по периферии листа неполных клеток (фиг.2), шт.;

$I_{S}$ - количество на изображении листа полных клеток (фиг. 1), шт.

Таблица 2

Количество клеток, периметр, площадь и расчетные параметры листа

\begin{tabular}{|c|c|c|c|c|c|c|c|c|c|c|}
\hline \multirow[b]{2}{*}{$\begin{array}{c}\text { № } \\
\text { П/П }\end{array}$} & \multicolumn{10}{|c|}{29.05 .2014} \\
\hline & $\begin{array}{l}I_{P}, \\
\text { шт. }\end{array}$ & $\begin{array}{l}I_{S}, \\
\text { Шт. }\end{array}$ & $\begin{array}{l}P, \\
\mathrm{cM}\end{array}$ & $\begin{array}{c}S, \\
\mathrm{~cm}^{2}\end{array}$ & $\begin{array}{l}K \\
\mathrm{~cm}\end{array}$ & $k$ & $\begin{array}{c}a, \\
\text { MM }\end{array}$ & $\begin{array}{l}b, \\
\text { MM }\end{array}$ & $k_{0}$ & $\vartheta$ \\
\hline 1 & 74 & 591 & 20,93 & 25,12 & 1,2002 & 0,0573 & 54 & 68 & 0,6841 & 0,7941 \\
\hline 2 & 112 & 624 & 31,68 & 27,20 & 0,8586 & 0,0271 & 62 & 60 & 0,7312 & 1,0333 \\
\hline 3 & 58 & 602 & 16,40 & 25,24 & 1,5386 & 0,0938 & 62 & 60 & 0,6785 & 1,0333 \\
\hline 4 & 58 & 532 & 16,40 & 22,44 & 1,3679 & 0,0834 & 60 & 62 & 0,6032 & 0,9677 \\
\hline 5 & 55 & 596 & 15,56 & 24,94 & 1,6032 & 0,1031 & 58 & 50 & 0,8600 & 1,1600 \\
\hline 6 & 67 & 664 & 18,95 & 27,90 & 1,4723 & 0,0777 & 58 & 56 & 0,8590 & 1,0357 \\
\hline 7 & 82 & 685 & 23,19 & 29,04 & 1,2521 & 0,0540 & 52 & 46 & 1,2140 & 1,1304 \\
\hline 8 & 70 & 639 & 19,80 & 26,96 & 1,3617 & 0,0688 & 60 & 54 & 0,8321 & 1,1111 \\
\hline 9 & 80 & 765 & 22,63 & 32,20 & 1,4231 & 0,0629 & 62 & 62 & 0,8377 & 1,0000 \\
\hline 10 & 75 & 695 & 21,21 & 29,30 & 1,3812 & 0,0651 & 66 & 72 & 0,6166 & 0,9167 \\
\hline
\end{tabular}

Дополнительно к физическим вводят расчетные параметры листьев:

- показатель формы листа (см)

$$
K=S / P
$$

- коэффициент формы листа

$$
k=S / P^{2} ;
$$

- коэффициент прямоугольной формы (по аналогу)

$$
k_{0}=100 S /(a b) \text {; }
$$

- коэффициент удлиненности листа

$$
\vartheta=a / b .
$$

Данные измерений по двум датам позволяют сравнивать между собой две статистические выборки. 
Описательная статистика. Для биологических объектов описательная статистика основывается на понятии вариации. Вариация - различие значений какого-либо признака у разных единиц совокупности за один и тот же промежуток времени.

Причиной возникновения вариации являются различные условия существования (развития и роста) разных единиц совокупности. Вариация - необходимое условие существования и развития массовых явлений.

Определение вариации необходимо при организации выборочного наблюдения, статистическом моделировании и планировании экспертных опросов. По степени вариации можно судить об однородности совокупности, устойчивости значений признака, типичности средней, о взаимосвязи между какимилибо признаками.

Абсолютные показатели вариации. Размах $R$ статистической выборки определяется по формуле

$$
R=y_{\max }-y_{\min } .
$$

Размах вариации показывает признак колебаний от максимума до минимума, но не учитывает распределения признака между ними.

Среднее арифметическое $\bar{y}$ выборки определяется формулой

$$
\bar{y}=\frac{1}{n} \sum_{i=1}^{n} y_{i},
$$

где $i$ - номер члена статистического ряда, $n$ - количество членов статистического ряда, шт., $y_{i}$ - член статистического ряда.

Среднее линейное отклонение $a_{л}$ характеризует среднее отклонение по модулю индивидуальных значений признака от среднего уровня и определяется выражением

$$
a_{л}=\frac{1}{n} \sum_{i=1}^{n}\left|y_{i}-\bar{y}\right|
$$

Дисперсия $\sigma^{2}$ малой выборки относительно среднего арифметического значения показателя, при числе наблюдений менее 20 , исчисляется по формуле

$$
\sigma^{2}=\frac{1}{n-1} \sum_{i=1}^{n}\left(y_{i}-\bar{y}\right)^{2} .
$$

Среднеквадратичное отклонение $\sigma$ определяется так:

$$
\sigma=\sqrt{\sigma^{2}}
$$

Среднеквадратичное отклонение показывает абсолютное отклонение фактических (измеренных) значений показателя от среднеарифметического значения.

Относительные показатели вариации. Относительный размах $\rho$ вариации (коэффициент осцилляции) определяется по соотношению

$$
\rho=R / \bar{y} .
$$

Относительное отклонение $m$ по модулю (линейный коэффициент вариации) вычисляется из математического отношения

$$
m=a_{\Omega} / \bar{y} .
$$


Коэффициент вариации $V, \%$ (показатель изменчивости) статистической выборки будет определяться формулой

$$
V=100 \sigma / \bar{y} .
$$

Коэффициент вариации случайной величины - мера относительного разброса случайной величины; показывает, какую долю среднего значения этой величины составляет ее средний разброс.

В отличие от среднего квадратичного или стандартного отклонения измеряет не абсолютную, а относительную меру разброса значений признака в статистической совокупности. Исчисляется в процентах. Вычисляется только для количественных данных. Чем больше значение коэффициента вариации, тем относительно больший разброс и меньшая выравненность значений исследуемого показателя.

Показатель точности наблюдений вычисляется по формуле

$$
P=V / \sqrt{n} .
$$

Доверие к данным и количество наблюдений. Тогда доверительные интервалы изменения изучаемого показателя будут равны:

$$
y_{\min }^{\text {meop }}=\bar{y}-[t] \sigma ; \quad y_{\max }^{\text {meop }}=\bar{y}+[t] \sigma .
$$

Опыты необходимы с малым риском при погрешности менее 5\%, поэтому $[t]=1,96$. Для решения вопроса об исключения наблюдения необходимо выяснить сущность отклонения (так называемые «особые» точки).

Формально, если выполнено соотношение

$$
y_{\min } \geq y_{\min }^{\text {meop }} \text { и } y_{\max } \leq y_{\max }^{\text {meop }},
$$

то такое наблюдение оставляется в статистической совокупности.

Решающее значение при проведении моментных наблюдений имеет объем производимых наблюдений. Он должен быть достаточным, чтобы обеспечить достоверность и репрезентативность получаемых результатов.

Допустимое значение $[n]$ вычисляется по формулам:

- при слабом уровне требований к точности измерений

$$
n \geq[t] \sigma /[\Delta]=[n] ;
$$

- при среднем уровне требований к измерениям

$$
n \geq[t] \sigma^{2} /[\Delta]^{2}=[n]
$$

- при сильном уровне требований к измерениям

$$
n \geq[t]^{2} \sigma^{2} /[\Delta]^{2}=[n],
$$

где $\quad n$ - принимаемое для последующих опытов количество наблюдений в виде измеряемых на локальной зоне древесного растения листьев, шт.;

$[t]$ - нормированное отклонение ( $t$-сигмальная единица), которое равно 1,96 при 95\% доверительной вероятности или при риске 5\% опыта;

$\sigma$ - среднее квадратичное отклонение остатков от среднего арифметического значения физического или расчетного параметра листа;

$[\Delta]$ - предельно допустимая относительная ошибка выборки остатков от среднего арифметического значения параметра листа (5\%);

$[n]$ - предельно допустимое малое количество наблюдений, шт. 
Проведем расчет показателей описательной статистики для последующего обоснования необходимого объема выборки.

Распределение параметров листьев липы. Для обработки измеренных данных применяется метод среднего арифметического значения параметров листьев. Примем допустимую погрешность $|\Delta|=5 \%$ и $[t]=1,96$.

Результаты вычислений показаны в таблице 3 и таблице 4.

Таблица 3

Описательная статистика распределения физических параметров листьев липы

\begin{tabular}{|c|c|c|c|c|c|c|c|c|c|}
\hline \multirow{2}{*}{\multicolumn{2}{|c|}{ Статистические показатели }} & \multicolumn{4}{|c|}{21.05 .2014} & \multicolumn{4}{|c|}{ 29.05.2014 } \\
\hline & & $\begin{array}{c}a, \\
\text { MM }\end{array}$ & $\begin{array}{l}b, \\
\text { MM }\end{array}$ & $\begin{array}{l}P \\
\mathrm{~cm}\end{array}$ & $\begin{array}{c}S, \\
\mathrm{~cm}^{2}\end{array}$ & $\begin{array}{c}a, \\
\text { MM }\end{array}$ & $\begin{array}{l}b, \\
\text { MM }\end{array}$ & $\begin{array}{l}P \\
\mathrm{cM}\end{array}$ & $\begin{array}{c}S, \\
\mathrm{~cm}^{2}\end{array}$ \\
\hline \multicolumn{2}{|l|}{ Максимальное значение $y_{\max }$} & 60 & 60 & 18,95 & 25,18 & 66 & 72 & 31,68 & 32,20 \\
\hline \multicolumn{2}{|l|}{ Минимальное значение $y_{\text {mиn }}$} & 36 & 40 & 12,16 & 10,70 & 52 & 46 & 15,56 & 22,44 \\
\hline \multicolumn{2}{|l|}{ Размах выборки $R$} & 24 & 20 & 6,79 & 14,48 & 14 & 26 & 16,12 & 9,76 \\
\hline \multicolumn{2}{|l|}{ Среднее арифметическое $\bar{y}$} & 50,4 & 48,8 & 15,16 & 19,24 & 59,4 & 59,0 & 20,68 & 27,63 \\
\hline \multicolumn{2}{|c|}{ Среднее линейное отклонение $a_{л}$} & 5,60 & 5,76 & 2,07 & 3,80 & 3,12 & 6,00 & 3,25 & 2,09 \\
\hline \multicolumn{2}{|c|}{ Дисперсия $\sigma^{2}$ выборки } & 54,04 & 50,84 & 5,85 & 22,53 & 16,93 & 61,56 & 22,01 & 7,67 \\
\hline \multicolumn{2}{|c|}{ Среднеквадратичное отклонение $\sigma$} & 7,351 & 7,131 & 2,420 & 4,747 & 4,115 & 7,846 & 4,691 & 2,769 \\
\hline \multicolumn{2}{|c|}{ Относительный размах $\rho$} & 0,467 & 0,409 & 0,448 & 0,753 & 0,235 & 0,440 & 0,779 & 0,361 \\
\hline \multicolumn{2}{|l|}{ Относительное отклонение $m$} & 0,111 & 0,118 & 0,137 & 0,198 & 0,052 & 0,101 & 0,157 & 0,077 \\
\hline \multicolumn{2}{|c|}{ Коэффициент вариации $V, \%$} & 14,59 & 14,61 & 15,96 & 24,67 & 6,93 & 13,30 & 22,68 & 10,24 \\
\hline \multicolumn{2}{|c|}{ Показатель точности наблюдений $P, \%$} & 4,61 & 4,62 & 5,05 & 7,80 & 2,19 & 4,21 & 7,17 & 3,24 \\
\hline \multicolumn{2}{|c|}{ Теоретическое максимум $y_{\max }^{\text {meop }}$} & 65 & 63 & 19,90 & 28,54 & 67 & 74 & 29,87 & 32,46 \\
\hline \multicolumn{2}{|l|}{ Теоретическое минимум $y_{\min }^{\text {meop }}$} & 36 & 35 & 10,42 & 9,94 & 51 & 44 & 11,49 & 21,60 \\
\hline \multirow{3}{*}{$\begin{array}{l}\text { Допустимое количество } \\
\text { наблюдений }[n] \text { при уровне } \\
\text { требовании: }\end{array}$} & слабом & 3 & 3 & 1 & 2 & 2 & 3 & 2 & 1 \\
\hline & среднем & 4 & 4 & 1 & 2 & 2 & 5 & 2 & 1 \\
\hline & сильном & 8 & 8 & 1 & 4 & 3 & 10 & 4 & 1 \\
\hline
\end{tabular}

Из данных таблицы 3 видно, что наилучшим параметром становится площадь поверхности листа. С увеличением возраста листочка требуется всего один лист без всяких повторов.

Периметр с возрастом листа увеличивает количество наблюдений от одного в самом молодом возрасте 19 дней (вегетационный период начался 2 мая).

Длина листа также имеет свойство стабилизировать свое значение в два раза (на среднем уровне требований к измерениям от 4 до 2).

А ширина листа, наоборот, с возрастом получает более высокую изменчивость значений (от 4 повторов наблюдений до 5).

Для дальнейших опытов все четыре параметра листа становятся надежными. Поэтому принимаем линейные и площадные параметры листа, по ним для 95\% доверительной вероятности при среднем уровне требований к измерениям нужны 1-5 листочков. Эксперименты дальше будем проводить при пяти повторах.

Сравнение физических и расчетных параметров показывает, что наибольшую надежность при методе среднего арифметического имеет параметры 
$S$ (площадь листа) и $\vartheta$ (коэффициент удлиненности листа). Оба они со временем вегетации снижаются.

Таблица 4

Описательная статистика расчетных параметров формы листьев липы

\begin{tabular}{|c|c|c|c|c|c|c|c|c|}
\hline \multirow[b]{2}{*}{ Статистические показатели } & \multicolumn{4}{|c|}{21.05 .2014} & \multicolumn{4}{|c|}{29.05 .2014} \\
\hline & $\begin{array}{l}K, \\
\mathrm{~cm}\end{array}$ & $k$ & $k_{0}$ & $\vartheta$ & $\begin{array}{l}K, \\
\mathrm{~cm}\end{array}$ & $k$ & $k_{0}$ & $\vartheta$ \\
\hline Максимальное значение $y_{\max }$ & 1,8024 & 0,1416 & 0,8771 & 1,2500 & 1,6032 & 0,1031 & 1,2140 & 1,1600 \\
\hline Минимальное значение $y_{\text {тип }}$ & 0,7138 & 0,0476 & 0,6965 & 0,7931 & 0,8586 & 0,0271 & 0,6032 & 0,7941 \\
\hline Размах выборки $R$ & 1,0886 & 0,0940 & 0,1806 & 0,4569 & 0,7446 & 0,0760 & 0,6108 & 0,3659 \\
\hline Среднее арифметическое $\bar{y}$ & 1,2919 & 0,0887 & 0,7746 & 1,0439 & 1,3459 & 0,0693 & 0,7916 & 1,0182 \\
\hline Среднее линейное отклонение $a_{л}$ & 0,3115 & 0,0299 & 0,0629 & 0,1370 & 0,1454 & 0,0161 & 0,1289 & 0,0789 \\
\hline Дисперсия $\sigma^{2}$ выборки & 0,1382 & 0,0012 & 0,0052 & 0,0270 & 0,0439 & 0,0005 & 0,0317 & 0,0117 \\
\hline Среднеквадратичное отклонение $\sigma$ & 0,3720 & 0,0341 & 0,0723 & 0,1642 & 0,2095 & 0,0217 & 0,1780 & 0,1082 \\
\hline Относительный размах $\rho$ & 0,843 & 1,060 & 0,234 & 0,441 & 0,553 & 1.097 & 0,772 & 0,359 \\
\hline Относительное отклонение $m$ & 0,241 & 0,337 & 0,081 & 0,131 & 0,108 & 0,132 & 0,163 & 0,077 \\
\hline Коэффициент вариации $V, \%$ & 28,79 & 38,44 & 9,33 & 15,72 & 15,57 & 31,31 & 22,49 & 10,63 \\
\hline Показатель точности наблюдений $P, \%$ & 9,10 & 12,16 & 2,95 & 4,97 & 4,92 & 9,90 & 7,11 & 3,36 \\
\hline Теоретическое максимум $y_{\max }^{\text {meop }}$ & 2,0210 & 0,1555 & 0,8591 & 1,2481 & 1,7565 & 0,1118 & 1,1405 & 1,2308 \\
\hline Теоретическое минимум $y_{\min }^{\text {meop }}$ & 0,5630 & 0,0219 & 0,6331 & 0,8397 & 0,9353 & 0,026 & 0,4427 & 0,8061 \\
\hline
\end{tabular}

Заключение. Преимуществом предлагаемого способа является техническая простота исполнения, так как из оборудования требуется только изготовить даже самому палетку, а сотовый телефон с функцией фотографирования ныне очень распространен и имеется почти у каждого студента или школьника. Кроме того, преимуществом является расчет по двум датам измерений параметров листов необходимого для статистической надежности количества листов, которые следует в опытах измерять с одной локальной зоны в кроне древесных растений.

Поэтому техническое решение может быть широко реализовано в школьных экологических кружках, пришкольных лесничествах, и даже в детских садах, а также в любых географических и иных экспедициях. При этом дополнительное введение периметра листа позволяет повысить точность измерений и площади листа.

\section{Литература}

1. Федорова А.И., Никольская А.Н. Практикум по экологии и охране окружающей среды: учеб. пос. М.: Гуманит. изд. центр ВЛАДОС, 2001. 288 с.

2. Пат. 2466351 Российская Федерация, МПК G 01 В 5 / 26 (2006.01). Способ измерения площади листьев у древесных растений / Мазуркин П.М., Щербакова Э.С.; заявитель и патентообладатель Поволжский. гос. технолог. ун-т. №2011123352/28; заявл. 08.06.2011; опубл. 10.11.2012.

3. Мазуркин П.М., Щербакова Э.С. Биотехническая закономерность встречаемости листьев у деревьев в различных экологических зонах города // Казанская наука. Казань: Изд-во Казанский Изд. Дом, 2011. № 1. С. 21-24. 
4. Мазуркин П.М. Коррелятивная вариация: учеб. пос. с грифомУМО РАЕ. Йошкар-Ола: Поволжский ГТУ, 2013. 120 с.

5. Мазуркин П.М. Статистическое моделирование. Эвристикоматематический подход. Йошкар-Ола: МарГТУ, 2001. 100с. 